\title{
Extracorporeal Shock Wave Therapy Ameliorates Hindlimb Ischemia in Rabbits
}

\author{
Keij Oi, ${ }^{1}$ Yoshiniro Funumoto, ${ }^{1,2}$ Kenta Ito, ${ }^{2}$ Toyokazu Uwatoku, ${ }^{1}$ \\ Kohtaro Abe, ${ }^{1}$ Takatoshi Hizume ${ }^{1}$ and Hiroaki ShimoKawa ${ }^{1,2}$ \\ ${ }^{1}$ Department of Cardiovascular Medicine, Kyushu University Graduate School of Medical \\ Sciences, Fukuoka, Japan \\ ${ }^{2}$ Department of Cardiovascular Medicine, Tohoku University Graduate School of Medicine, \\ Sendai, Japan
}

\begin{abstract}
We have recently demonstrated that the low-energy extracorporeal cardiac shock wave (SW) therapy improves myocardial perfusion and cardiac function in a porcine model of chronic myocardial ischemia and also ameliorates myocardial ischemia in patients with severe coronary artery disease. The present study was designed to examine whether our SW therapy also is effective to ameliorate hindlimb ischemia in rabbits. Hindlimb ischemia was made by surgical excision of the entire unilateral rabbit femoral artery. One week after the operation, we performed the SW $(n=9)$ or sham-therapy $(n=9)$ to the ischemic region 3 times a week for 3 weeks. Three weeks after the SW therapy, the development of collateral arteries, the flow ratio of the ischemic/non-ischemic common iliac arteries, the blood pressure ratio of the ischemic/non-ischemic hindlimb, and the capillary density in the ischemic muscles were all significantly increased in the SW group compared with the control group, indicating that the SW therapy induced therapeutic angiogenesis. Importantly, no adverse effect, such as muscle damage, hemorrhage, or thrombosis, was noted with the therapy. Finally, we examined the role of endothelial nitric oxide synthesis (eNOS) and vascular endothelial growth factor (VEGF) in the mechanisms of SW-induced angiogenesis on day 28. The expression levels of eNOS and VEGF proteins in ischemic hindlimb muscles tended to be increased in the SW group compared with the control group. These results suggest that our low-energy SW therapy also is effective and safe for the treatment of peripheral artery disease. — shock wave therapy; angiogenesis; peripheral artery disease; blood flow; capillary density.
\end{abstract}

Tohoku J. Exp. Med., 2008, 214 (2), 151-158.

(C) 2008 Tohoku University Medical Press

The severity of peripheral arterial disease (PAD) is closely associated with the risk of myocardial infarction, ischemic stroke, and death from vascular causes (Caro et al. 2005). The current management of PAD has three major therapeutic options, including medical treatment, percutane- ous transluminal angioplasty, and bypass surgery. However, prognosis of patients with severe PAD still remains poor when there is no indication of bypass surgery or percutaneous transluminal angioplasty (Hirsch et al. 2006). Angiogenesis is a new promising therapeutic strategy for severe

Received December 14, 2007; revision accepted for publication January 18, 2008.

Correspondence: Yoshihiro Fukumoto, M.D., Ph.D., Department of Cardiovascular Medicine, Tohoku

University Graduate School of Medicine, 1-1 Seiryo-machi, Aoba-ku, Sendai 980-8575, Japan.

e-mail: fukumoto@cardio.med.tohoku.ac.jp 
PAD, however, gene or cell therapy is invasive in nature, difficult to repeat, and still at preclinical stage (Isner et al. 1996; Leschke et al. 1996; Losordo et al. 1998; Tateishi-Yuyama et al. 2002).

We have recently demonstrated that lowenergy shock wave (SW) could induce various angiogenic factors and enhance angiogenesis in vitro (Nishida et al. 2004). SW is a longitudinal acoustic wave, traveling with the speed in water of ultrasound through body tissue, which is a single pressure pulse with a short needle-like positive spike of less than 1 microsecond duration and amplitude up to $100 \mathrm{MPa}$ followed by a tensile part of several microseconds with lower amplitude (Apfel 1982). SW is known to exert "cavitation effect" (a micrometer sized violent collapse of bubbles inside the cells) and has recently been demonstrated to induce localized stress on cell membranes that resembles shear stress (Apfel 1982; Maisonhaute et al. 2002). We have recently demonstrated that low-energy extracorporeal SW therapy effectively induces angiogenesis and ameliorates myocardial perfusion and cardiac function in a porcine model of chronic myocardial ischemia and in patients with severe coronary artery disease without any adverse effects (Nishida et al. 2004; Fukumoto et al. 2006; Uwatoku et al. 2007). Furthermore, it has been recently demonstrated that low-energy SW therapy improves recruitment of circulating endothelial progenitor cells via enhanced expression of chemoattractant factors in hindlimb ischemia of rat model (Aicher et al. 2006). The present study was thus designed to examine whether our SW therapy also is effective to ameliorate severe hindlimb ischemia in rabbits.

\section{METHODS}

All procedures were approved by the Institutional Animal Care and Use Committee and were conducted in conformity with the institutional guidelines of Kyushu University. The authors had full access to the data and take full responsibility for its integrity. All authors have read and agree to the article as written.

\section{Animal preparation}

Male New Zealand White rabbits (weight, 3.0 to 3.2 $\mathrm{kg}$ ) were anesthetized with an intramuscular injection of xylazine $(5 \mathrm{mg} / \mathrm{kg})$ and ketamine $(50 \mathrm{mg} / \mathrm{kg})$. Rabbit unilateral hindlimb ischemia model was made as previously described (Takeshita et al. 1994). Briefly, under sterile surgical conditions, we performed a longitudinal incision in the left hindlimb, where the femoral artery was completely excised from its proximal origin to the point where it bifurcates into the popliteal and saphenous arteries including all branches of the femoral artery (Takeshita et al. 1994).

\section{Extracorporeal cardiac SW therapy}

One week after the induction of left hindlimb ischemia, we performed the SW therapy $\left(0.09 \mathrm{~mJ} / \mathrm{mm}^{2}\right.$, about $10 \%$ of the energy for lithotripsy treatment, 30 spots in the ischemic muscle, 200 shots/spot) (Nishida et al. 2004) to the animals with a guidance of ultrasonic echogram 3 times a week for 3 weeks $(n=9)$. In the previous study, we confirmed that the current energy level of shock wave exerts maximum angiogenic effects) (Nishida et al. 2004).

\section{Study protocol}

The SW therapy was performed 3 times a week, starting at day 7 for 3 weeks, while the control group received the same procedures 3 times a week without the SW therapy ( $n=9$ each). Angiography was performed at day 7 and 28, and blood flow, histological, and immunoblotting analyses were done at day 28 of sacrifice.

\section{Angiographic analysis}

Selective angiography of the ischemic limb was performed at day 7 and 28 after the surgery ( $n=6$ each), using the quantitative cineangiography (QCA) system (Toshiba Medical, Tokyo) (Nishida et al. 2004; Uwatoku et al. 2007). A $4 \mathrm{~F}$ end-hole infusion catheter was inserted into the left common iliac artery of the ischemic limb via the common carotid artery. Then, angiography was performed with $4 \mathrm{~mL}$ of contrast media $(1 \mathrm{~mL} / \mathrm{sec})$ for $8 \mathrm{sec}$. To quantitatively assess collateral vessel development, a grid with 2.5-mm-diameter squares was placed over the angiogram in the region of middle thigh. The number of squares crossed by contrast-pacified arteries was counted and angiographic score was calculated as the ratio of the number of total squares (Nishida et al. 2004; Uwatoku et al. 2007). To quantitatively assess collateral vessel flow, the number of frame from beginning of infusion of contrast media to filling to distal popliteal and saphenous artery were calculated by recorded angiography (Nishida et al. 2004; Uwatoku et al. 2007). 


\section{Common iliac artery blood flow ratio}

Common iliac artery blood flow was directly measured by ultrasonic transit-time flow meter (Transonic Systems Inc, NYC, NY, USA) at the time of sacrifice (day $28, n=9$ each). We performed a longitudinal incision in the lower abdomen, where lower abdominal aorta and bilateral common iliac artery was carefully isolated, and bilateral common iliac artery blood flow was simultaneously measured at least for $5 \mathrm{~min}$. The ratio of ischemic to non-ischemic hindlimb blood flow was defined in each rabbit as the ratio of mean common iliac artery flow ( $\mathrm{ml} /$ min) of the ischemic limb to that of the non-ischemic limb.

\section{Lower limb calf blood pressure ratio}

Calf blood pressure was measured with auto-detected blood pressure monitor (BP100D, Fukuda ME Kogyo Co., Ltd., Tokyo) at day $28(n=9$, each). The ratio of ischemic to non-ischemic hindlimb blood flow was defined for each rabbit as the ratio of systolic blood pressure $(\mathrm{mmHg})$ of the ischemic limb to that of the nonischemic limb.

\section{Lower limb muscle weight}

We measured the weight of the adductor muscle and the semimembranous muscle of the ischemic and nonischemic limbs of each animal ( $n=7-8$ each) at the time of sacrifice (day 28).

\section{Capillary density and capillary /muscle fiber ratio}

Tissue specimens were obtained as transverse sections from the adductor muscle and the semimembranous muscle of the ischemic limb of each animal ( $n=6$ each) at the time of sacrifice (day 28). Muscle samples were embedded in OCT compound and snap-frozen in liquid nitrogen. Multiple frozen sections were then sliced (5 $\mu \mathrm{m}$ in thickness) from each specimen on a cryostat (Nishida et al. 2004; Uwatoku et al. 2007). The tissue sections were stained for alkaline phosphatase using an indoxyl-tetra-zolium method to detect capillary endothelial cells, as previously described, and were counterstained with eosin (Nishida et al. 2004; Uwatoku et al. 2007). A total of 20 different fields from the two muscle were randomly selected, and capillaries were counted under a x100 objective to determine the capillary density (number of capillaries $/ \mathrm{mm}^{2}$ ) (Nishida et al. 2004; Uwatoku et al. 2007). In order to ensure that analysis of capillary density was not overestimated because of muscle atrophy, capillary density was also evaluated as a function of the number of muscle fibers in the section (capillaries/muscle fibers ratio) (Nishida et al. 2004; Uwatoku et al. 2007).

\section{Western blot analysis for eNOS and VEGF protein expression}

Whole cell proteins were extracted from the adductor muscle and the semimembranous muscle of the ischemic limb of each group ( $n=6$ each) at day 28. Mouse monoclonal antibody to human endothelial nitric oxide synthesis (eNOS, pS633, BD Transduction Laboratories, San Jose, CA, USA) and human vascular endothelial growth factor (VEGF, G143-850, BD Pharmingen, San Jose, CA, USA) were used. The same amount of extract protein $(50 \mu \mathrm{g})$ was loaded for SDS-PAGE/ immunoblotting analysis. The regions containing eNOS and VEGF proteins were visualized by ECL Western blotting luminal regent (Santa Cruz Biotechnology, Santa Cruz, CA, USA). The levels of Western blot for eNOS and VEGF were normalized to those for $\beta$-actin as an internal control.

\section{Statistical analysis}

Continuous variables were expressed as mean \pm S.E.M. Comparisons of all parameters were evaluated by unpaired $t$-test. All statistical analyses were performed using Stat View (SAS Institute, Cary, NC, USA), and P values of less than 0.05 were considered to be statistically significant.

\section{RESUlts \\ Hemodynamic variables \\ At day 7 and day 28 after the surgery, sys- temic arterial pressure and heart rate were compa- rable between the control and the SW groups (data not shown).}

\section{Angiogenic effects of the SW therapy}

The selective internal iliac angiography showed that both of control and SW-treated rabbits did not have well-developed collateral arteries at day 7 (Fig. 1A, C), while SW therapy increased the collateral fillings at day 28 as compared with the control group (Fig. 1B, D). The evaluation by angiographic score and filling time to distal popliteal and saphenous artery demonstrated that the SW therapy significantly increased the angiographic collateral fillings at day 28 (Fig. 2). 


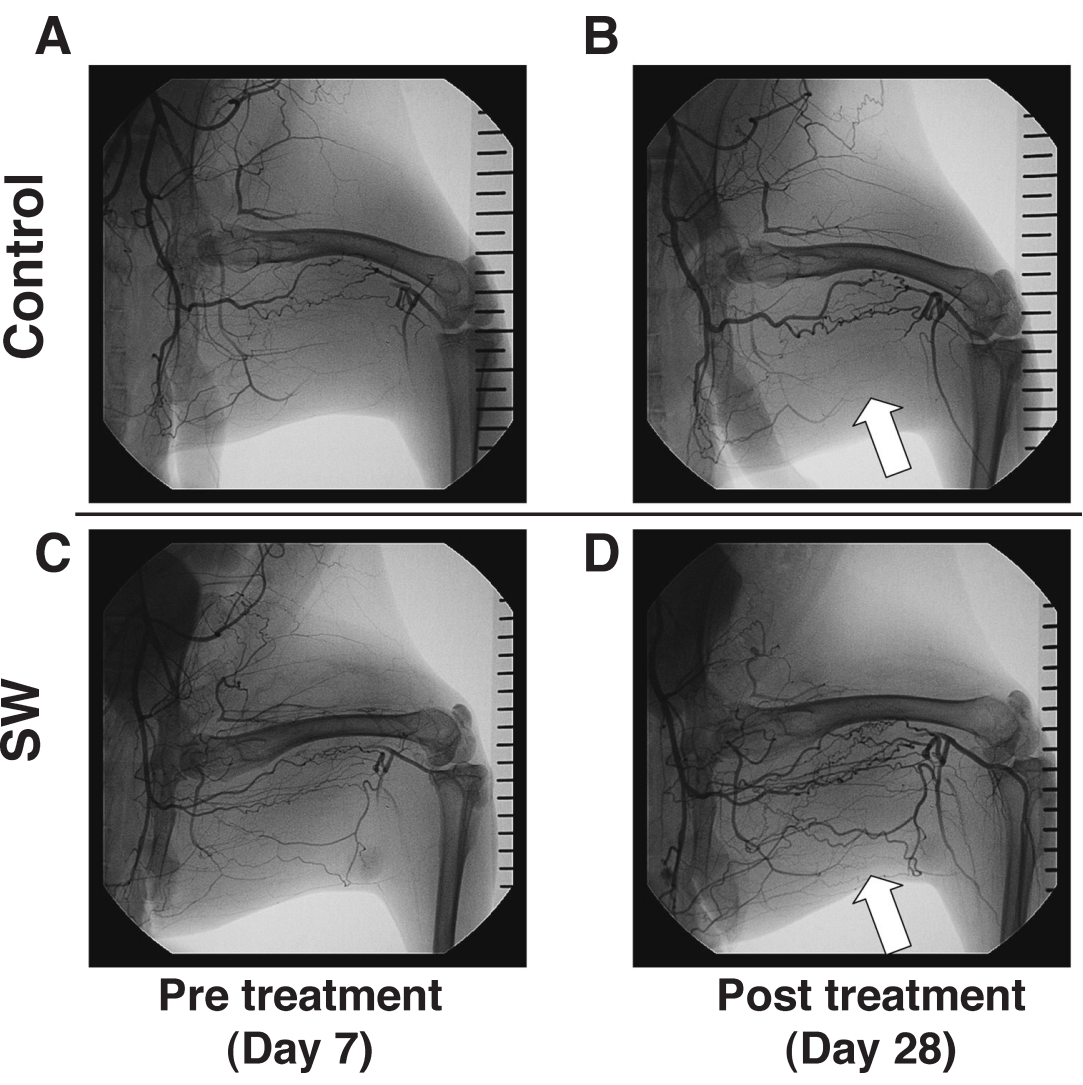

Fig. 1. Representative selective internal iliac angiography of control rabbit at day 7 (A) and day 28 (B), and shock wave (SW)-treated rabbit at day 7 (C, before SW therapy) and day 28 (D, 3 weeks after SW therapy). Note that increased collateral arteries were noticed in the SW-treated animal at day 28 (D).
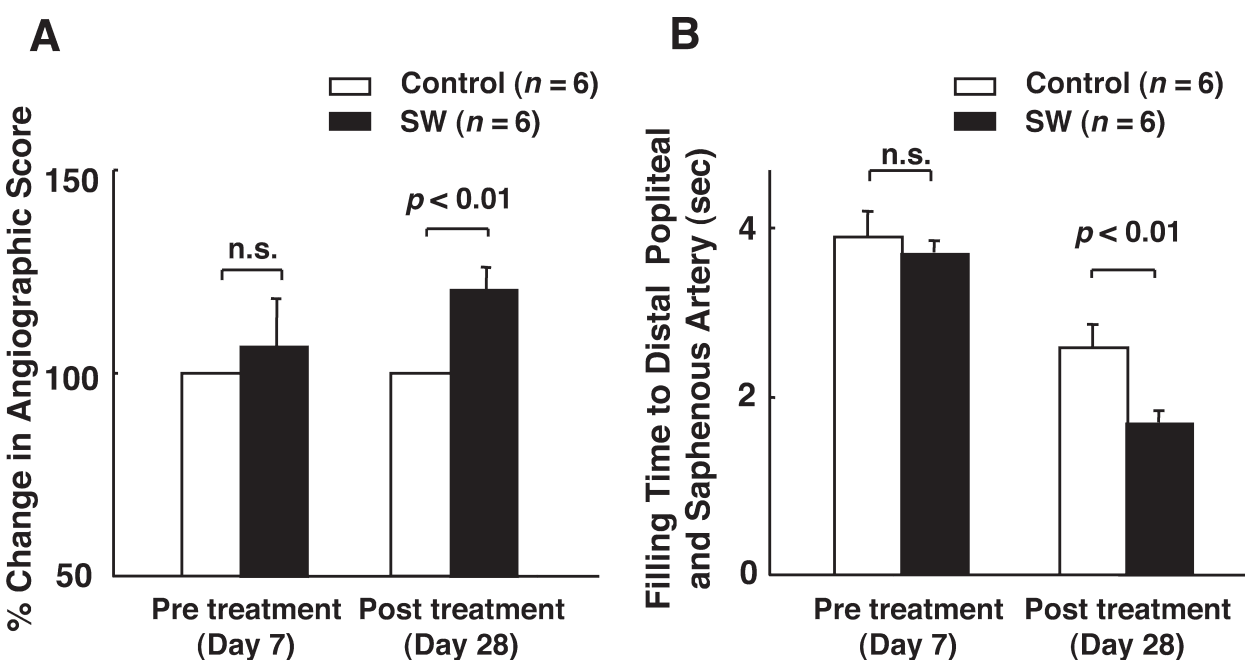

Fig. 2. Effects of the SW therapy in the ischemic limbs on collateral vessel growth and collateral blood flow by angiography. (A) The SW therapy significantly increased the angiographic score expressed as percent change compared with control group at day 28. (B) Filling time to distal popliteal and saphenous artery was significantly decreased in the SW group compared with the control group at day 28. Results are expressed as means \pm S.E.M. 
A

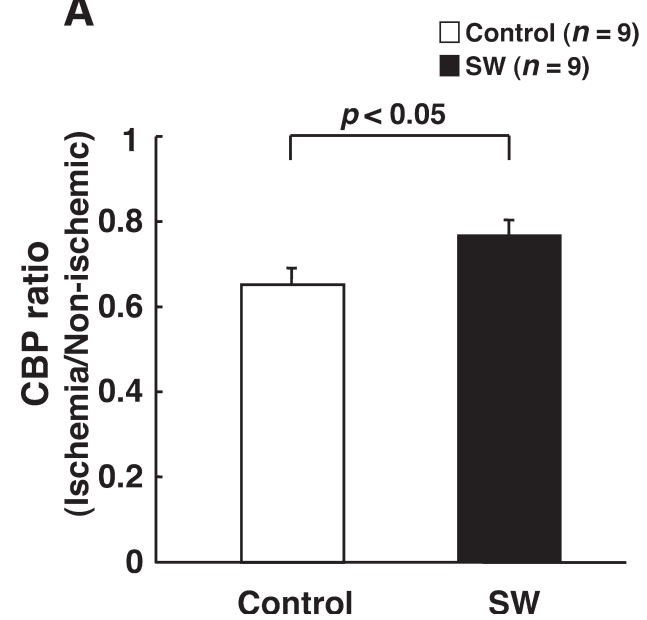

B

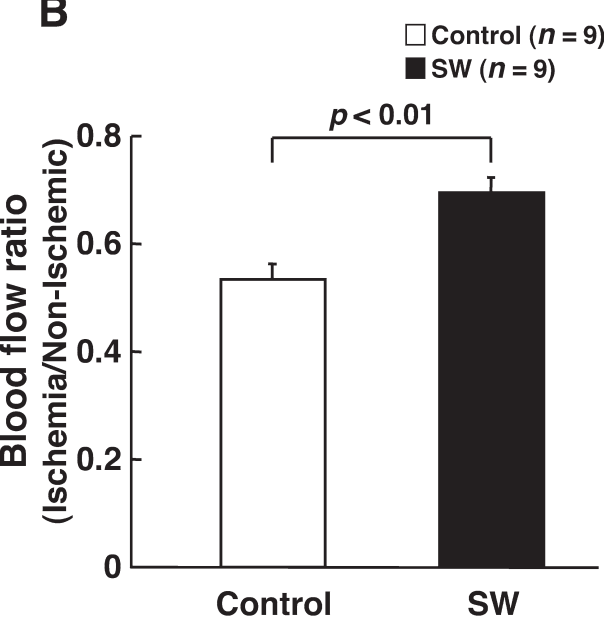

Fig. 3. Effects of the SW therapy in the ischemic limb on hindlimb blood pressure and common iliac arterial blood flow. The SW therapy significantly improved the calf blood pressure (CBP) ratio (ischemic/non-ischemic hindlimb) (A) and the common iliac flow ratio (ischemic/normal hindlimb) (B) at day 28. Results are expressed as means \pm S.E.M.

Furthermore, the SW therapy to the ischemic hindlimb significantly improved the calf blood pressure ratio and the common iliac flow ratio (ischemic/non-ischemic hindlimb) (Fig. 3). Although the SW therapy tended to increase the weight of the ischemic lower limbs, the effect did not reach the statistically significant level (Fig. 4). It increased capillary density in the ischemic muscle as compared with the control group at day 28 (Fig. 5). No adverse effect (e.g. muscle damage, hemorrhage, thrombosis) was noted with the SW therapy.

\section{Effects of the SW therapy on eNOS and VEGF expression}

The SW therapy tended to increase eNOS and VEGF protein expression in the ischemic limb at day 28 (Fig. 6).

\section{Discussion}

The novel finding of the present study is that our non-invasive SW therapy increased angiographic collaterals and blood flow in rabbits with ischemic limb without any procedural complications or adverse effects. The present result is in accordance with our previous findings that our non-invasive extracorporeal cardiac SW therapy ameliorates myocardial ischemia in a porcine

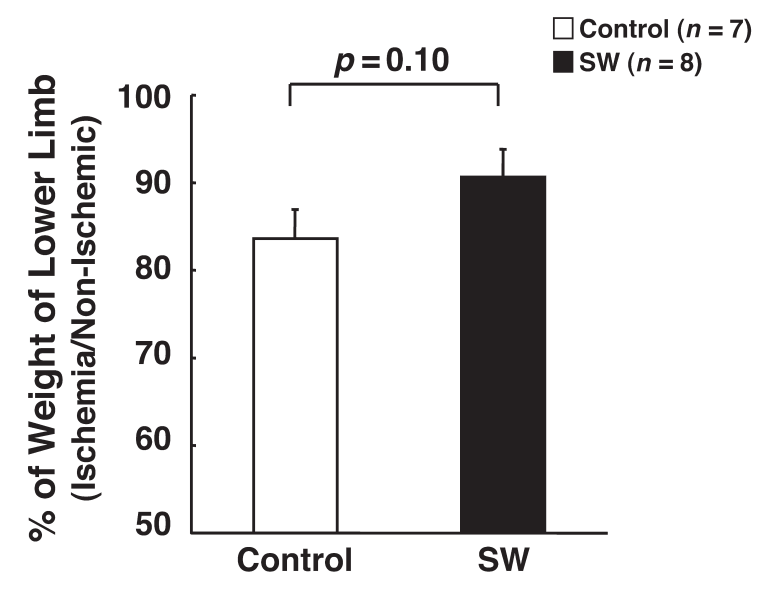

Fig. 4. Effects of the SW therapy in the ischemic/ non-ischemic limbs on the weight of the adductor muscle and the semimembranous muscle. The SW therapy tended to increase the weight of ischemic lower limb muscle at day 28. Results are expressed as means \pm S.E.M.

model of chronic myocardial ischemia and in patients with severe coronary artery disease (Nishida et al. 2004; Fukumoto et al. 2006; Uwatoku et al. 2007).

\section{Mechanism of the SW therapy-induced angio- genesis \\ The precise mechanism of a low-energy SW to induce angiogenesis remains to be fully eluci-}


A

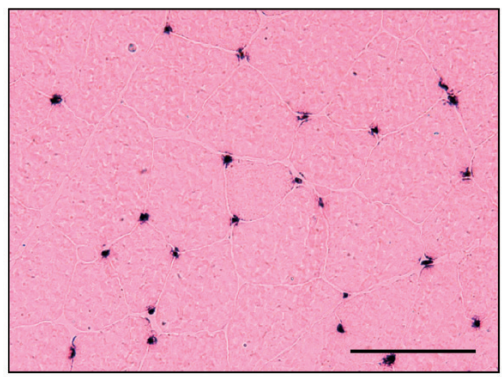

C

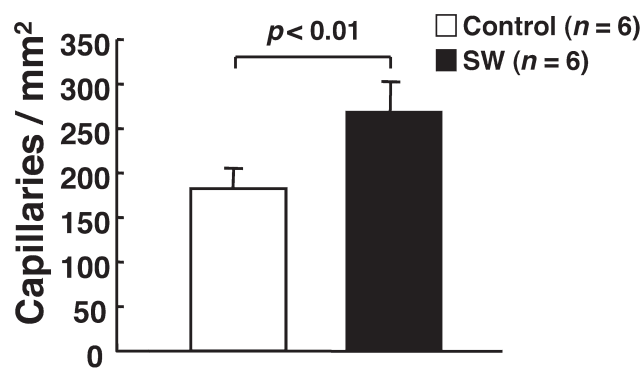

B

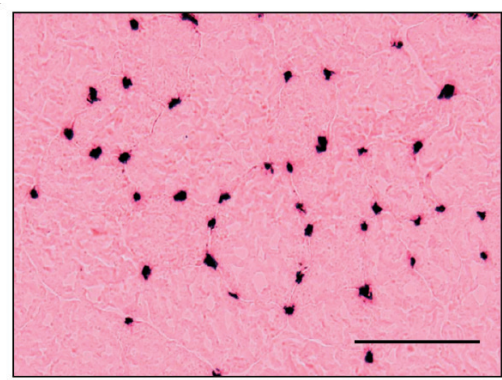

D

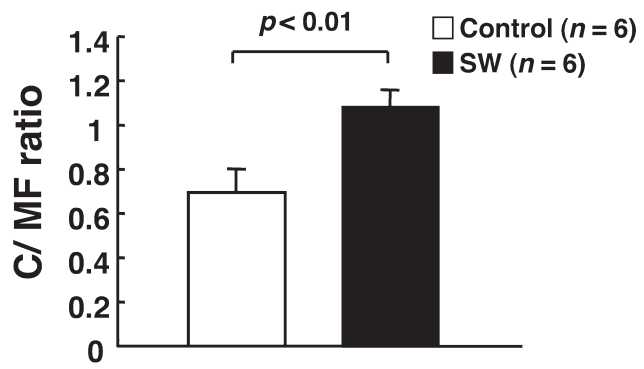

Fig. 5. Effects of the SW therapy on capillary density in the ischemic skeletal muscle. Capillary density in the ischemic muscle was increased in the SW group (B) compared with the control group (A) at day 28. Dark blue dots indicate capillaries that are positive for alkaline phosphatase staining. Bars indicate $100 \mu \mathrm{m}$. Quantitative analysis of capillary density indicates that capillaries $/ \mathrm{mm}^{2}$ (C) and capillaries/muscle fiber ratio (D) in the ischemic muscle were both significantly greater in the SW group than in the control group. Results are expressed as means \pm S.E.M.
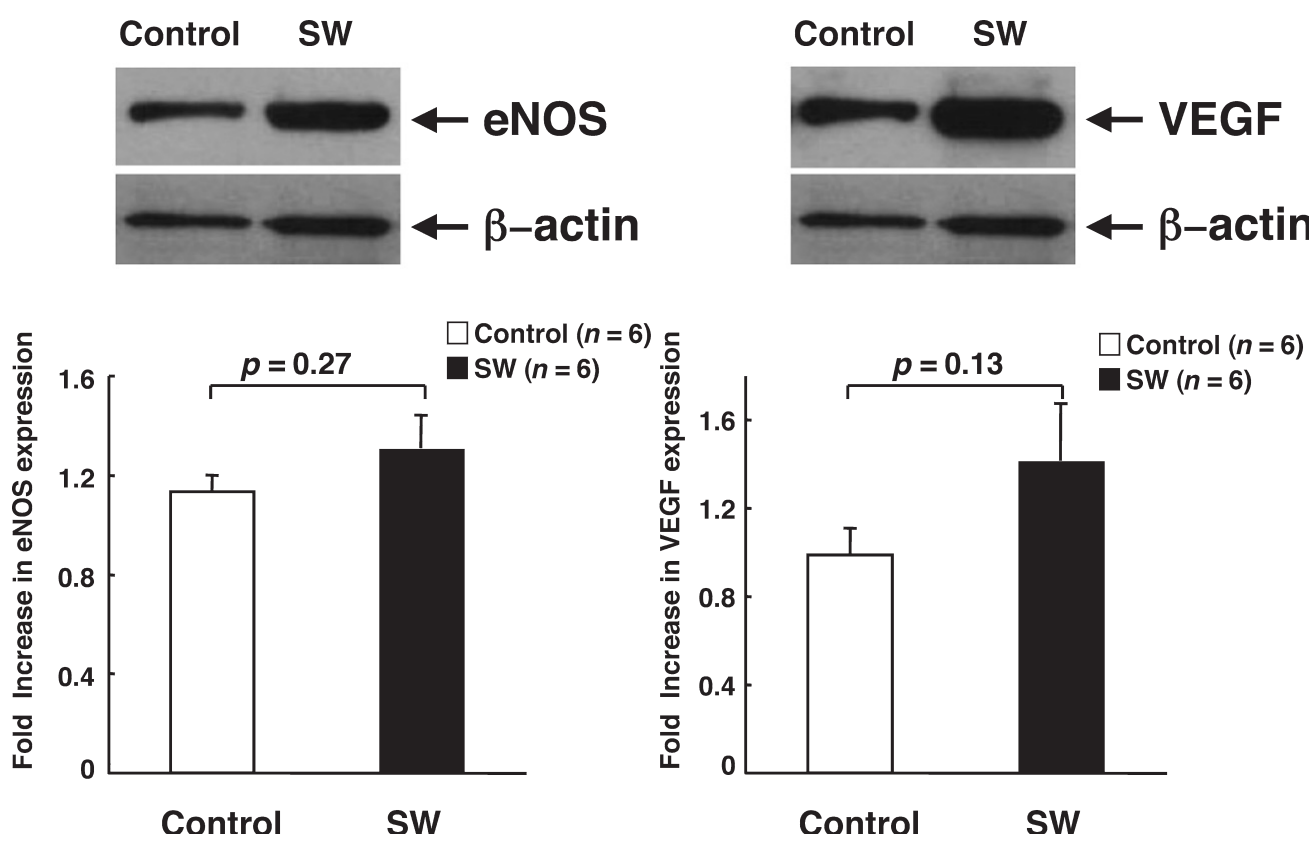

Fig. 6. Effects of the SW therapy on the protein expression of eNOS (A) and VEGF (B) in the ischemic skeletal muscle. The SW therapy tended to increase eNOS and VEGF expression in the ischemic limb. Results are expressed as means \pm S.E.M. 
dated; however, it has been demonstrated that SW can cause non-enzymatic nitric oxide (NO) synthesis from L-arginine and hydrogen peroxide (Gotte et al. 2002), and that SW induces neovascularization at tendon (Rompe et al. 1995; Wang et al. 2003) and up-regulates the expression of eNOS, VEGF, and proliferating cell antigen (PCNA) (Wang et al. 2003). We have previously confirmed that low-energy SW up-regulates VEGF and its receptor, Flt-2, in endothelial cells in vitro and VEGF in the ischemic porcine myocardium in vivo (Nishida et al. 2004). Recently, Aicher et al. (2006) have demonstrated that lowenergy SW up-regulates mRNA expression of the chemoattractant stromal cell-derived factor 1 and the number of VEGF-positive endothelial cells at various energy levels, resulting in significantly enhanced recruitment and homing of endothelial progenitors that were intravenously administered $24 \mathrm{hrs}$ after SW therapy in a rat model of hindlimb ischemia. However, in the present study, we were unable to demonstrate the significant up-regulation of eNOS or VEGF in the ischemic limb although there noted such a tendency. It is conceivable that the timing of the examination (day 28) might not be adequate to detect the up-regulation because the VEGF-Flt system could mainly affect the initiation of vasculogenesis and/or angiogenesis in ischemic limb (Nishida et al. 2004). It also is possible that endogenous angiogenic systems other than eNOS or VEGF are involved in the effect of the SW therapy. These points remain to be examined in future studies.

\section{Advantage of the non-invasive $S W$ therapy}

Although angiogenesis by gene or cell therapy may be effective in patients with severe PAD, it is invasive in nature and difficult to repeat (Isner et al. 1996; Leschke et al. 1996; Losordo et al. 1998; Tateishi-Yuyama et al. 2002). A major advantage of our SW therapy is shown by the fact that it is quite non-invasive and safe, without any procedural complications or adverse effects (Nishida et al. 2004; Fukumoto et al. 2006; Uwatoku et al. 2007). If necessary, we can repeatedly treat patients with our SW therapy because no surgery, anesthesia, or even catheter intervention is required for the therapy. This is an important factor in determining the clinical usefulness of angiogenic therapies in elderly patients with severe PAD. Therefore, our non-invasive SW therapy appears to be a useful treatment for ischemic PAD.

\section{Limitations of the study}

Several limitations should be mentioned for the present study. First, the present rabbit model lacks atherosclerotic lesions in the systemic or peripheral vasculature. Future studies are needed to demonstrate whether SW therapy is effective in animals or patients complicated with systemic and/or peripheral atherosclerosis. Second, as we discussed above, the up-regulation of eNOS and VEGF by the SW therapy at day 28 did not reach a statistically significant level. The precise mechanism of SW-induced angiogenesis remains to be examined in future studies. Third, it remains to be examined whether our SW therapy is also effective to ameliorate hindlimb ischemia with greater or lesser extent than that in the present rabbit model ( $\sim 50 \%$ reduction of blood flow). Fourth, the best strategy of SW delivery still remains to be elucidated. Our strategy was to shoot 200 shots/spot in 30 spots, 3 times a week for 3 weeks in the ischemic muscle in the present study (Nishida et al. 2004), while Aicher et al. (2006) have shot 500-2,000 shocks in one session. Further studies are required to determine the best treatment strategy. Finally, it was difficult to distinguish vasculogenesis from angiogenesis in this model, because we were unable to recognize which vessels were de novo formation of blood vessels and which were from pre-existing vascular network.

\section{Conclusions}

We were able to demonstrate that our lowenergy non-invasive SW therapy effectively induces angiogenesis in ischemic limbs, resulting in the increase in collateral flow, hindlimb blood pressure, and arterial blood flow without any adverse effects. Thus, our SW therapy could be a useful strategy for the treatment of PAD. 


\section{Acknowledgments}

This study was supported in part by the grantsin-aid and the grant for the 21 st Century COE Program from the Japanese Ministry of Education, Culture, Sports, and Technology and that from the Program for Promotion of Fundamental Studies in Health Sciences of the Organization for Pharmaceutical Safety and Research of Japan.

\section{References}

Aicher, A., Heeschen, C., Sasaki, K., Urbich, C., Zeiher, A.M. \& Dimmeler, S. (2006) Low-energy shock wave for enhancing recruitment of endothelial progenitor cells: a new modality to increase efficacy of cell therapy in chronic hind limb ischemia. Circulation, 114, 2823-2830.

Apfel, R.E. (1982) Acoustic cavitation: a possible consequence of biomedical uses of ultrasound. Br. J. Cancer Suppl., 45, 140-146.

Caro, J., Migliaccio-Walle, K., Ishak, K.J. \& Proskorovsky, I. (2005) The morbidity and mortality following a diagnosis of peripheral arterial disease: long-term follow-up of a large database. BMC Cardiovasc. Disord., 5, 14.

Fukumoto, Y., Ito, A., Uwatoku, T., Matoba, T., Kishi, T., Tanaka, H., Takeshita, A., Sunagawa, K. \& Shimokawa, H. (2006) Extracorporeal cardiac shock wave therapy ameliorates myocardial ischemia in patients with severe coronary artery disease. Coron Artery Dis., 17, 63-70.

Gotte, G., Amelio, E., Russo, S., Marlinghaus, E., Musci, G. \& Suzuki, H. (2002) Short-time non-enzymatic nitric oxide synthesis from L-arginine and hydrogen peroxide induced by shock waves treatment. FEBS Lett., 520, 153-155.

Hirsch, A.T., Haskal, Z.J., Hertzer, N.R., Bakal, C.W., Creager, M.A., Halperin, J.L., Hiratzka, L.F., Murphy, W.R., Olin, J.W., Puschett, J.B., Rosenfield, K.A., Sacks, D., Stanley, J.C., Taylor, L.M., Jr., White, C.J., White, J., White, R.A., Antman, E.M., Smith, S.C., Jr., Adams, C.D., Anderson, J.L., Faxon, D.P., Fuster, V., Gibbons, R.J., Halperin, J.L., Hiratzka, L.F., Hunt, S.A., Jacobs, A.K., Nishimura, R., Ornato, J.P., Page, R.L. \& Riegel, B. (2006) ACC/AHA 2005 guidelines for the management of patients with peripheral arterial disease (lower extremity, renal, mesenteric, and abdominal aortic): executive summary a collaborative report from the American Association for Vascular Surgery/Society for Vascular Surgery, Society for Cardiovascular Angiography and Interventions, Society for Vascular Medicine and Biology, Society of Interventional Radiology, and the ACC/AHA Task Force on Practice Guidelines (Writing Committee to Develop Guidelines for the Management of Patients With Peripheral Arterial Disease) endorsed by the American Association of Cardiovascular and Pulmonary Rehabilitation; National Heart, Lung, and Blood Institute; Society for Vascular Nursing; TransAtlan- tic Inter-Society Consensus; and Vascular Disease Foundation. J. Am. Coll. Cardiol., 47, 1239-1312.

Isner, J.M., Pieczek, A., Schainfeld, R., Blair, R., Haley, L., Asahara, T., Rosenfield, K., Razvi, S., Walsh, K. \& Symes, J.F. (1996) Clinical evidence of angiogenesis after arterial gene transfer of phVEGF165 in patient with ischaemic limb. Lancet, 348, 370-374.

Leschke, M., Schoebel, F.C., Mecklenbeck, W., Stein, D., Jax, T.W., Muller-Gartner, H.W. \& Strauer, B.E. (1996) Longterm intermittent urokinase therapy in patients with endstage coronary artery disease and refractory angina pectoris: a randomized dose-response trial. J. Am. Coll. Cardiol., 27, 575-584.

Losordo, D.W., Vale, P.R., Symes, J.F., Dunnington, C.H., Esakof, D.D., Maysky, M., Ashare, A.B., Lathi, K. \& Isner, J.M. (1998) Gene therapy for myocardial angiogenesis: initial clinical results with direct myocardial injection of phVEGF165 as sole therapy for myocardial ischemia. Circulation, 98, 2800-2804.

Maisonhaute, E., Prado, C., White, P.C. \& Compton, R.G. (2002) Surface acoustic cavitation understood via nanosecond electrochemistry. Part III: Shear stress in ultrasonic cleaning. Ultrason Sonochem, 9, 297-303.

Nishida, T., Shimokawa, H., Oi, K., Tatewaki, H., Uwatoku, T., Abe, K., Matsumoto, Y., Kajihara, N., Eto, M., Matsuda, T., Yasui, H., Takeshita, A. \& Sunagawa, K. (2004) Extracorporeal cardiac shock wave therapy markedly ameliorates ischemia-induced myocardial dysfunction in pigs in vivo. Circulation, 110, 3055-3061.

Rompe, J.D., Rumler, F., Hopf, C., Nafe, B. \& Heine, J. (1995) Extracorporal shock wave therapy for calcifying tendinitis of the shoulder. Clin. Orthop. Relat. Res., 321, 196-201.

Takeshita, S., Pu, L.Q., Stein, L.A., Sniderman, A.D., Bunting, S., Ferrara, N., Isner, J.M. \& Symes, J.F. (1994) Intramuscular administration of vascular endothelial growth factor induces dose-dependent collateral artery augmentation in a rabbit model of chronic limb ischemia. Circulation, 90, II228-II234.

Tateishi-Yuyama, E., Matsubara, H., Murohara, T., Ikeda, U., Shintani, S., Masaki, H., Amano, K., Kishimoto, Y., Yoshimoto, K., Akashi, H., Shimada, K., Iwasaka, T. \& Imaizumi, T. (2002) Therapeutic angiogenesis for patients with limb ischaemia by autologous transplantation of bonemarrow cells: a pilot study and a randomised controlled trial. Lancet, 360, 427-435.

Uwatoku, T., Ito, K., Abe, K., Oi, K., Hizume, T., Sunagawa, K. \& Shimokawa, H. (2007) Extracorporeal cardiac shock wave therapy improves left ventricular remodeling after acute myocardial infarction in pigs. Coron Artery Dis., 18, $397-404$

Wang, C.J., Wang, F.S., Yang, K.D., Weng, L.H., Hsu, C.C., Huang, C.S. \& Yang, L.C. (2003) Shock wave therapy induces neovascularization at the tendon-bone junction. A study in rabbits. J. Orthop. Res., 21, 984-989. 